\title{
Encoded physics knowledge in checking codes for nuclear cross section libraries at Los Alamos
}

\author{
D. Kent Parsons ${ }^{\mathrm{a}}$ \\ Nuclear Data Team, Group XCP-5, Los Alamos National Laboratory, USA
}

\begin{abstract}
Checking procedures for processed nuclear data at Los Alamos are described. Both continuous energy and multi-group nuclear data are verified by locally developed checking codes which use basic physics knowledge and common-sense rules. A list of nuclear data problems which have been identified with help of these checking codes is also given.
\end{abstract}

\section{Introduction}

Nuclear data libraries used by transport codes are produced by nuclear data processing codes like NJOY from evaluated nuclear data files. Quality assurance (or verification) of the nuclear data is performed by checking codes at several points during the generation of the evaluated data and the processing of the data. The last stage of nuclear data checking performed at Los Alamos is the subject of this paper.

Nuclear cross section libraries produced by NJOY and maintained by the Nuclear Data Team at Los Alamos are either in ACE (continuous energy) or NDI (multigroup) format. The final check of ACE files at Los Alamos is performed by the locally developed and maintained CHECKACE code. The final check of NDI tables at Los Alamos is performed by the locally developed and maintained CHECKMG code. Both of these programs encode basic physics knowledge to check the data in nuclear cross section libraries.

\section{Checking of continuous energy ACE files at Los Alamos}

CHECKACE $[1,2]$ is a collection of FORTRAN and PERL routines which uses common sense physics-based rules and known MCNP limitations to check the data in ACE files. The intent of CHECKACE is to identify errors in the ACE data and or to document unusual data values which might be indications of other problems. CHECKACE is a brain-dead but very useful tool which is used by the nuclear data team to verify ACE files. It does not - and indeed cannot - catch every conceivable ACE file error, but it quickly checks for many possible errors.

The capabilities of CHECKACE have recently been extended by the addition of new rules and the modification of previous rules. Since the rules are logical expression of physics knowledge, they are the real core capability of CHECKACE. In fact, many of the rules of CHECKACE

a e-mail: dkp@lanl.gov were developed in the aftermath of and as a consequence of nuclear data problems which have been encountered. Hence, over time, the rule checking becomes more comprehensive and effective.

\subsection{Some rules of CHECKACE}

First, CHECKACE verifies that the sum of the partial cross sections is equal to the total cross section (within a given tolerance) at every neutron energy in the energy grid given in the first block of data in the ACE file. It checks if any of the other partial cross sections which go into the total cross section are negative. Negative cross sections are unphysical.

CHECKACE performs similar checks for the photon production cross sections (total $=$ the sum of the partials within a tolerance and no negative values). It also checks if the interpolated midpoint values of the total cross sections and partial cross section sums are within a tolerance for both neutron cross sections and photon production cross sections. This midpoint coding in CHECKACE assumes linear-linear interpolation and thus it will produce small errors if something other than linear-linear interpolation is present in the ACE file.

CHECKACE also checks if the prompt and total nu values and also the MT 5 neutron production values are non-negative and also not too large. Large is defined as 9.0. The reason for this rule is the MCNP limitation of eleven (11) secondary particles from any given reaction.

The pdf/cdf values for various distributions in the ACE files produced (or transcribed) by NJOY are checked for negative pdf values (which are unphysical) and also checked to verify that the cdf values are monotonic and that the last cdf value is 1.0. This checking now includes all scattering reaction angular distributions. Since NJOY sets any negative PDF values it finds to very small positive values, CHECKACE looks for these very small PDF values. These values may be indicative of a negative scattering problem (e.g., a truncated Legendre expansion

(C) The Authors, published by EDP Sciences. This is an Open Access article distributed under the terms of the Creative Commons Attribution License 4.0 (http://creativecommons.org/licenses/by/4.0/). 
that goes negative at some values) in the evaluated nuclear data.

\section{Checking of multi-group NDI files at Los Alamos}

CHECKMG [3] is a C code which checks NJOY produced multi-group neutron cross sections in NDI format. It uses NDI calls to read the multi-group data and employs a set of physics-based common sense rules to check the data. Analogous to CHECKACE, the intent of CHECKMG is to identify errors in the NDI data and or to document unusual data values which might be indications of other problems. CHECKMG is also brain-dead but it is a very useful tool which is used by the nuclear data team to verify NDI files. It does not - and indeed cannot - catch every conceivable NDI file error, but it quickly checks for many possible errors.

CHECKMG has recently been extended to encode more physics-based knowledge by the addition of more rules and the modification of previous rules. Analogous to CHECKACE, these rules are the core capability of CHECKMG and are continually developed as new data problems are encountered.

\subsection{Some rules of CHECKMG}

First, the sum of the partial cross sections is compared with the total cross section for every neutron group. Negative cross sections are flagged since they are unphysical. The cross section balance of the Legendre scattering matrix is also checked - i.e., the neutron production from each incoming neutron energy group in the matrix is compared with the sum of the individual neutron producing reactions for that incoming energy - including the multiplicity (e.g., 1.0 for scattering, 2.0 for $n, 2 n$ reactions, etc.). Fission chi normalizations to 1.0 are verified. Values of nu are checked for negativity as well as for being within reasonable bounds.

CHECKMG looks for negative $\mathrm{P}_{0}$ cross sections in the Legendre scattering matrix - since physics and mathematics require such values to be positive (or zero). The mathematics of Legendre expansions also requires (and CHECKMG verifies) that the absolute value of the $\mathrm{P}_{1}(1=1, \mathrm{~L})$ cross sections must be less than or equal to the corresponding $\mathrm{P}_{0}$ value.

CHECKMG checks the consistency of kerma and heating values - since kerma $=$ heating $*$ sigtot. It also verifies that the NJOY provided kerma value is within a tolerance of the kerma value calculated from the sum of all of the reaction cross sections* their $\mathrm{Q}$ values. The values of $\mathrm{Q}$ for each reaction are also verified as being reasonable.

\section{Some results from nuclear data team checking codes}

In isotopic order, here are some of the nuclear data anomalies identified and fixed with the help the Nuclear Data Team checking codes, CHECKACE and CHECKMG. An incorrect set of $\mathrm{H}-1$ gamma production CDF's were identified by CHECKACE and a numerical precision bug in NJOY was fixed. An incomplete cross section balance in B-10 was spotted by CHECKMG and a problem in the local NDIR module for NJOY was identified. Negative scattering in B-11 was identified by both CHECKACE and CHECKMG which led to some corrections of the high energy scattering cross sections. An incorrect number placement in the $\mathrm{Cl}-35$ evaluation file was indirectly indicated by CHECKMG and fixed in the evaluation. Problems in the evaluation files for Co-58 MT 103 and Eu-153 low energy gamma production were found by CHECKMG and CHECKACE. A bug in the NDIR module of NJOY with regards to fission gamma production was indicated by CHECKMG and has since been fixed. Finally, problems in the MT 2 cross sections for U-237 and U-239 are flagged by CHECKMG and have led to a change in the official Los Alamos versions of the evaluation files.

There are a few cases where the nuclear data problem is still outstanding and has not yet been fixed. An inconsistency in the Np-239 gamma production was identified by CHECKMG, while a problem in the MT 5 neutron production for $\mathrm{Pa}$ is flagged by CHECKACE.

\section{Summary}

Physics-based common sense rules undergird the nuclear data library checking codes used at Los Alamos. Checking codes like CHECKACE and CHECKMG are now officially a part of the standard nuclear data processing and verification procedures used by the nuclear data team in group XCP-5 at Los Alamos.

\section{References}

[1] D.K. Parsons, "The Rules of CHECKACE - a Suite of Checking Codes for MCNP ACE Cross Section Files", Memo XCP-5:15-032, Los Alamos National Laboratory (2015)

[2] D.K. Parsons, "More Rules of CHECKACE - a Suite of Checking Codes for MCNP ACE Cross Section Files", Memo XCP-5:17-002, Los Alamos National Laboratory (2016)

[3] D.K. Parsons and J.M. Campbell, "The Rules of CHECKMG - a checking code for NDI XS Data Tables", Memo XCP-5:11-008, Los Alamos National Laboratory (2011) 